\section{Nature's semantics}

lan Stewart

Complexity: The Emerging Science at the Edge of Order and Chaos. By M. Mitchell Waldrop. Simon and SchusterNiking: 1992. Pp. 380. \$23 (hbk), £9.99 (pbk).

Complexity: Life at the Edge of Chaos. By Roger Lewin. Macmillan, New YorkDent: 1993. Pp. 208. \$22, £15.99.

NEW buzzwords are flying, and both authors manage to get all of them into their title and subtitle: 'complexity', the "edge of chaos'. Heady stuff. "Vital and controversial", says Stephen Jay Gould in the blurb for Lewin's book; "a deep tale of science in the making", says Douglas Hofstadter on Waldrop's. Is complexity theory really novel and exciting? Does either book capture its idiosyncratic and slightly elusive character?

In brief: yes and yes. The complexity theorists have an important message to convey; and although similar messages have come down to us from previous ages, notably at the urging of some philosophers of science, this particular version of the message locks in more directly with problems of genuine concern for real scientists. An idea mustn't just arrive: everybody has to know it's arrived. And both books manage to explain the main concerns and conclusions of the complexity theorists without getting tangled up in fine points. Lewin's book is more journalistic, more of a 'people' book; Waldrop's goes into slightly more depth on the science. Both focus on the Santa Fe Institute in New Mexico, as the first well-known centre specially set up to study complexity (but let us not forget Stephen Wolfram's Centre for Complex Systems). Both leave us wanting to know more, which is the proper function of a popular science book. Both, for journalistic reasons, tell the story from one viewpoint, the complexity theorists', and fail to ask some awkward questions. But what they tell, they tell well; and anyone not brain-dead or hopelessly reactionary should listen.

Complexity, in the sense used here, is not mere complication. It is that curious mix of complication and organization that we find throughout the natural and human worlds: the workings of a cell, the structure of the brain, the behaviour of the stock market, the shifts of political power. The underlying thesis of complexity theory is that traditional reductionism - understanding systems by breaking them down into components and analysing the interactions between them - cannot provide an adequate understanding of such systems. It is the old problem of 'emergent phenomena', of wholes that somehow transcend their parts - indeed of wholes that are to a great extent immune to the detailed structure of their parts. It is about as far as you can get from the 'theory of everything' philosophy espoused by many cosmologists and particle physicists; because - to put it crudely - it holds that stock markets would still crash if the laws of quantum chromodynamics were completely different.

The crux of the argument is that there are three kinds of system: those that don't do anything terribly interesting (steady states, periodic cycles - a lot of classical physics, in short); those that are so complicated that they are just a huge mess, lacking even interesting statistical features (gas molecules, most of the rest of classical physics); and those that hover in between, structured but unpredictable, a flux of almost-patterns (most of biology, economics, politics, sociology, psychology). The three horsemen of the dynamic apocalypse: Death, Chaos and Complexity. Or Death, Chaos and Life, as it is living systems above all that occupy the middle ground, the mysterious 'edge of chaos'.

The common character of complex systems is the interaction of large numbers of reasonably similar components; but the viewpoint is that various largescale phenomena emerge naturally and predictably within such a context. Many of those phenomena are baffling to any reductionist view: they include selforganization, increasing complication and sophistication, unexpectedly long periods of quiescence, sudden bursts of wild activity. Despite - maybe because of - an immense reductionist attack on the molecular structure and function of DNA, we really know very little indeed about biological development: about form rather than chemistry. As Richard Lewontin recently wrote in The New York Review of Books (28 May 1992), the common image of DNA as a selfreplicating molecule is about as true as describing a letter as a self-replicating document. The letter needs a photocopier; the DNA needs a cell. Perhaps we should occasionally look at how the photocopier works.

It is not sensible in a short review to try to summarize the arguments for and against this position. The issues are themselves complex: on the one hand, many precursors for such thoughts exist, so the theory can be dismissed as 'nothing new'. On the other hand, if enough scientists went along with it, it would strike at the heart of much orthodoxy. Traditional mathematical economics, for example, with its emphasis on equilibrium and 'rational expectations', would largely be wiped out. This may well be one of the core arguments in favour of complexity theory, because economic events do seem inconsistent with the classical models.

Scientists of a more traditional bent that is, most of us - would do well to take serious note of the kinds of question that complexity theory hammers home. They should be much less serious about its current answers to those questions. In particular the 'edge of chaos' is at best a partial and metaphorical answer to emergence. I note in passing that our metaphors in this area are getting very confused: 'chaos' used to mean total disorder; then, in the hands of what are now called chaos theorists, it came to mean apparent disorder with a simple, nonrandom cause; but in complexity theory is has once again become total disorder. Plus ça change.

I also think the mechanism of 'antichaos' - that emergence results from the narrowing of the range of possible internal states due to adaptation to the environment - is highly questionable. Emergence is surely more a matter of coherent large-scale function riding on and irreducibly driven by an incredibly intricate internal structure, but one that is 'transparent' to the large-scale function. Think of the human visual system. Our brains construct an amazingly effective model of reality, not because their internal structure has become simplified as a result of adaptation to the task at hand, but because their 'programming' has become inordinately complicated, even though its workings are necessarily hidden from our conscious minds. We can teach computers antichaos, but we can't teach them to see.

The importance of complexity theory, at this early stage in its development, lies in how it shifts the scientific goalposts away from ever-more-detailed analysis of fine internal structure towards a more global explanation of forms, features and functions. Both books convey the message clearly and readably: it is time science relearned how to look outwards as well as inwards, to think about meaning as well as counting information, and to appreciate nature's semantics as well as its syntax. This is the core of the complexity manifesto. Read it, think about it, disagree with it, make television programmes about it, write polemics against it — but don't ignore it.

Ian Stewart is in the Mathematics Institute, University of Warwick, Coventry CV4 $7 A L, U K$ 